Korean J. Math. 19 (2011), No. 1, pp. 1-16

\title{
ON A CERTAIN CLASS OF $p$-VALENT UNIFORMLY CONVEX FUNCTIONS USING DIFFERENTIAL OPERATOR
}

\author{
S. K. LeE, S. M. Khairnar and S. M. RAJAS
}

\begin{abstract}
In this paper, using differential operator, we have introduce new class of p-valent uniformly convex functions in the unit $\operatorname{disc} U=\{z:|z|<1\}$ and obtain the coefficient bounds, extreme bounds and radius of starlikeness for the functions belonging to this generalized class. Furthermore, partial sums $f_{k}(z)$ of functions $f(z)$ in the class $S^{*}(\lambda, \alpha, \beta)$ are considered. The various results obtained in this paper are sharp.
\end{abstract}

\section{Introduction}

Let $S$ denote the class of functions of the form

$$
f(z)=z^{p}+\sum_{n=p+1}^{\infty} a_{n} z^{n}, \quad a_{n} \geq 0, \quad(p \in N)
$$

which are analytic and p-valent in the unit disc $U=\{z:|z|<1\}$. Also $S^{*}$ denote the subclass of $S$ consisting of functions of the form

$$
f(z)=z^{p}-\sum_{n=p+1}^{\infty} a_{n} z^{n}, \quad a_{n} \geq 0, \quad(p \in N) .
$$

Received November 4, 2010. Revised March 4, 2011. Accepted March 10, 2011. 2000 Mathematics Subject Classification: 30C45, 30C50.

Key words and phrases: uniformly convex functions, coefficient bounds, extreme points, radius of starlikeness and partial sums.

The second author is thankful and acknowledges the support from the research projects funded by the Department of Science and Technology(DST), (Ref. No. SR/S4/MS:544/2008), Goverment of India, NBHM, Department of Atomic Energy, (Ref. No. NBHM/DAE/RP.2/09), Goverment of India, BCUD( University of Pune) and UGC New Delhi, India (Ref. No.47-992/09/(WRO)). 
G. Murugusundaramoorthy([1]), Goodman([3], [4]) and Ronning([5], [6]) have studied the following subclasses

i) A function $f(z) \in S$ is said to be in the class $S_{p}(\alpha, \beta)$ of uniformly $\beta$-starlike function if it satisfies the condition

$$
\operatorname{Re}\left\{1+\frac{z f^{\prime \prime}(z)}{f^{\prime}(z)}-\alpha\right\}>\beta\left|\frac{z f^{\prime}(z)}{f(z)}-1\right| \quad(z \in U),
$$

where $-1<\alpha \leq 1, \beta \geq 0$ and $p \in N$.

ii) A function $f(z) \in S$ is said to be in the class $U C V(\alpha, \beta)$ of uniformly $\beta$-convex function if it satisfies the condition

$$
\operatorname{Re}\left\{1+\frac{z f^{\prime \prime}(z)}{f^{\prime}(z)}-\alpha\right\}>\beta\left|\frac{z f^{\prime \prime}(z)}{f^{\prime}(z)}\right| \quad(z \in U)
$$

where $-1<\alpha \leq 1, \beta \geq 0$ and $p \in N$. It follows from (1.3) and (1.4) that $f(z) \in U C V(\alpha, \beta)$ is equivalent to

$$
z f^{\prime}(z) \in S_{p}(\alpha, \beta) \text {. }
$$

For the function $f(z) \in S$ is given by (1.1) and $g(z) \in S$ is given by, $g(z)=z^{p}+\sum_{n=p+1}^{\infty} b_{n} z^{n}$. We define the Hadamard product(convolution) of $f(z)$ and $g(z)$ given by,

$$
(f * g)(z)=z^{p}+\sum_{n=p+1}^{\infty} a_{n} b_{n} z^{n} \quad(z \in U, \quad p \in N) .
$$

For the function $f(z) \in S$, we define the following,

$$
I^{0} f(z)=f(z), \quad I^{1} f(z)=z f^{\prime}(z)+\frac{1+p}{z^{p}}
$$

and for $k=2,3, \cdots$

$$
I^{k} f(z)=z\left(I^{k-1} f(z)\right)^{\prime}+\frac{1+p}{z^{p}}=z^{p}+\sum_{n=p+1}^{\infty} n(k) a_{n} z^{n} \quad(p \in N),
$$


where $I^{k}$ is called as differential operator. Ghanim and Darus([2]) have studied this operator extensively.

Let $S^{*}(\alpha, \beta)$ be the subclass of $S$ consisting of the functions of the form (1.1) and satisfying the condition

$$
\left|\frac{\frac{z\left(I^{k} f(z)\right)^{\prime}}{I^{k} f(z)}-p}{\frac{\beta z\left(I^{k} f(z)\right)^{\prime}}{I^{k} f(z)}-\alpha p}\right|<\mu \quad(z \in U, \quad p \in N),
$$

where $-1 \leq \alpha<\beta \leq 1$ and $0<\mu \leq 1$. Also let $S^{* *}(\alpha, \beta)=S^{*}(\alpha, \beta) \cap$ $S^{*}$.

The main object of this paper is to study the coefficient estimates, extreme points and radius of starlikeness for the functions belonging to the generalized class $S^{* *}(\alpha, \beta)$. Furthermore, partial sums $f_{k}(z)$ of functions $f(z)$ in the class $S^{*}(\alpha, \beta)$ are considered and sharp lower bounds for the ratios of real part of $f(z)$ to $f_{k}(z)$ and $f^{\prime}(z)$ to $f_{k}^{\prime}(z)$ are determined.

In this paper, all the investigated results are motivated by Ron$\operatorname{ning}([5],[6])$, K. G. Subramanian ([10], [11]).

\section{Basic properties}

In this we obtain a necessary and sufficient condition for functions $f(z)$ in the classes $S^{*}(\alpha, \beta)$ and $S^{* *}(\alpha, \beta)$.

Theorem 2.1. A function $f(z)$ of the form (1.1) is in $S^{*}(\alpha, \beta)$ if

$$
\sum_{n=p+1}^{\infty}[(n-p)+\mu(n \beta-\alpha p)] n(k)\left|a_{n}\right| \leq \mu p(\beta-\alpha),
$$

where $-1 \leq \alpha<\beta \leq 1,0<\mu \leq 1$ and $p \in N$.

proof. Since $f(z) \in S^{*}(\alpha, \beta)$, it is sufficient to show that

$$
\left|\frac{\frac{z\left(I^{k} f(z)\right)^{\prime}}{I^{k} f(z)}-p}{\frac{\beta z\left(I^{k} f(z)\right)^{\prime}}{I^{k} f(z)}-\alpha p}\right|<\mu .
$$


We have

$$
\begin{aligned}
& \left|\frac{\frac{z\left(I^{k} f(z)\right)^{\prime}}{I^{k} f(z)}-p}{\frac{\beta z\left(I^{k} f(z)\right)^{\prime}}{I^{k} f(z)}-\alpha p}\right|=\left|\frac{\frac{p z^{p}+\sum_{n=p+1}^{\infty} n(k) a_{n} n z^{n}}{z^{p}+\sum_{n=p+1}^{\infty} n(k) a_{n} z^{n}}-p}{\frac{\beta\left\{p z^{p}+\sum_{n=p+1}^{\infty} n(k) a_{n} n z^{n}\right\}}{z^{p}+\sum_{n=p+1}^{\infty} n(k) a_{n} z^{n}}-\alpha p}\right| \\
& \leq\left|\frac{p z^{p}+\sum_{n=p+1}^{\infty} n(k) a_{n} n z^{n}-p z^{p}-p \sum_{n=p+1}^{\infty} n(k) a_{n} z^{n}}{\beta\left\{p z^{p}+\sum_{n=p+1}^{\infty} n(k) a_{n} n z^{n}\right\}-\alpha p\left[z^{p}+\sum_{n=p+1}^{\infty} n(k) a_{n} z^{n}\right]}\right| \\
& \leq\left|\frac{\sum_{n=p+1}^{\infty}(n-p) n(k) a_{n} n}{\beta p-\alpha p+\sum_{n=p+1}^{\infty}(n \beta-\alpha p) n(k) a_{n}}\right| \leq \mu .
\end{aligned}
$$

Allowing value of $z$ tends to 1 minus on the real axis, we get

$$
\sum_{n=p+1}^{\infty}[(n-p)+\mu(n \beta-\alpha p)] n(k)\left|a_{n}\right| \leq \mu p(\beta-\alpha) .
$$

THEOREM 2.2. A necessary and sufficient condition for $f(z)$ of the form (1.2) to be in the $S^{* *}(\alpha, \beta)$

$$
\sum_{n=p+1}^{\infty}[(n-p)+\mu(n \beta-\alpha p)] n(k)\left|a_{n}\right| \leq \mu p(\beta-\alpha),
$$

where $-1 \leq \alpha<\beta \leq 1,0<\mu \leq 1$ and $p \in N$.

Proof. In view of theorem (2.1), we need only to prove that necessity. If $f(z) \in S^{* *}(\alpha, \beta)$ and $z$ is real then

$$
\left|\frac{\frac{z\left(I^{k} f(z)\right)^{\prime}}{I^{k} f(z)}-p}{\frac{\beta z\left(I^{k} f(z)\right)^{\prime}}{I^{k} f(z)}-\alpha p}\right|<\mu .
$$

We have

$$
\left|\frac{\frac{z\left(I^{k} f(z)\right)^{\prime}}{I^{k} f(z)}-p}{\frac{\beta z\left(I^{k} f(z)\right)^{\prime}}{I^{k} f(z)}-\alpha p}\right| \leq\left|\frac{\frac{p z^{p}-\sum_{n=p+1}^{\infty} n(k) a_{n} n z^{n}}{z^{p}-\sum_{n=p+1}^{\infty} n(k) a_{n} z^{n}}-p}{\frac{\beta\left\{P z^{p}-\sum_{n=p+1}^{\infty} n(k) a_{n} n z^{n}\right\}}{z^{p}-\sum_{n=p+1}^{\infty} n(k) a_{n} z^{n}}-\alpha p}\right| .
$$


The above expression is bounded by $\mu$ then we obtain the inequality

$$
\sum_{n=p+1}^{\infty}[(n-p)+\mu(n \beta-\alpha p)] n(k)\left|a_{n}\right| \leq \mu p(\beta-\alpha),
$$

where $-1 \leq \alpha<\beta \leq 1,0<\mu \leq 1$ and $p \in N$.

In the following theorem we show that the class $S^{* *}(\alpha, \beta)$ is closed under convex linear combination.

Theorem 2.3. Let $f(z)$ defined by (1.2) and $g(z)=z^{p}-\sum_{n=p+1}^{\infty} b_{n} z^{n}$ be in the class $S^{* *}(\alpha, \beta)$. Then the function

$$
h(z)=(1-\xi) f(z)+\xi g(z)=z^{p}-\sum_{n=p+1}^{\infty} \eta_{n} z^{n}
$$

is also in the class $S^{* *}(\alpha, \beta)$, where $\eta_{n}=(1-\xi) a_{n}+\xi b_{n}, \quad 0 \leq \xi<1$.

Proof. Since the function $f(z)$ and $g(z)$ belongs to $S^{* *}(\alpha, \beta)$, we have

$$
\sum_{n=p+1}^{\infty}[(n-p)+\mu(n \beta-\alpha p)] n(k)\left|a_{n}\right| \leq \mu p(\beta-\alpha)
$$

and

$$
\sum_{n=p+1}^{\infty}[(n-p)+\mu(n \beta-\alpha p)] n(k)\left|b_{n}\right| \leq \mu p(\beta-\alpha) .
$$

Clearly,

$$
\begin{aligned}
h(z) & =(1-\xi) f(z)+\xi g(z) \\
& =(1-\xi)\left(z^{p}-\sum_{n=p+1}^{\infty} a_{n} z^{n}\right)+\xi\left(z^{p}-\sum_{n=p+1}^{\infty} b_{n} z^{n}\right) \\
& =z^{p}-\sum_{n=p+1}^{\infty}\left[(1-\xi) a_{n}+\xi b_{n}\right] z^{n} \\
& =z^{p}-\sum_{n=p+1}^{\infty} c_{n} z^{n}
\end{aligned}
$$


where $c_{n}=(1-\xi) a_{n}+\xi b_{n}$. Using (2.3) and (2.4),

$$
\begin{aligned}
\sum_{n=p+1}^{\infty}[ & (n-p)+\mu(n \beta-\alpha p)] n(k) c_{n} \\
= & \sum_{n=p+1}^{\infty}[(n-p)+\mu(n \beta-\alpha p)] n(k)\left[(1-\xi) a_{n}+\xi b_{n}\right] \\
= & (1-\xi) \sum_{n=p+1}^{\infty}[(n-p)+\mu(n \beta-\alpha p)] n(k) a_{n} \\
& \quad+\xi \sum_{n=p+1}^{\infty}[(n-p)+\mu(n \beta-\alpha p)] n(k) b_{n} \\
= & (1-\xi) \mu p(\beta-\alpha)+\xi \mu p(\beta-\xi) \\
\leq & \mu p(\beta-\alpha) .
\end{aligned}
$$

Thus we have $\sum_{n=p+1}^{\infty}[(n-p)+\mu(n \beta-\alpha p)] n(k) c_{n} \leq \mu p(\beta-\alpha)$. Hence $h(z) \in S^{* *}(\alpha, \beta)$.

Theorem 2.4(Extreme Points). Let $f_{1}(z)=z^{p}$ and for $n=$ $2,3,4, \cdots$

$$
f_{n}(z)=z^{p}-\frac{\mu p(\beta-\alpha)}{[(n-p)+\mu(n \beta-\alpha p)] n(k)} z^{n}
$$

Then $f(z) \in S^{* *}(\alpha, \beta)$ if and only if $f(z)$ can be expressed in the form $f(z)=\sum_{n=1}^{\infty} \xi_{n} f_{n}(z)$, where $\xi_{n} \geq 0$ and $\sum_{n=1}^{\infty} \xi_{n}=1$.

Proof. Suppose that $f(z)=\sum_{n=1}^{\infty} \xi_{n} f_{n}(z)$, then

$$
f(z)=z^{p}-\sum_{n=p+1}^{\infty} \frac{\mu p(\beta-\alpha)}{[(n-p)+\mu(n \beta-\alpha p)] n(k)} \xi_{n} z^{n}=z^{p}-\sum_{n=p+1}^{\infty} c_{n} z^{n}
$$


where $c_{n}=\frac{\mu p(\beta-\alpha)}{[(n-p)+\mu(n \beta-\alpha p)] n(k)} \xi_{n}$. Thus

$$
\begin{aligned}
& \sum_{n=p+1}^{\infty}[(n-p)+\mu(n \beta-\alpha p) n(k)] c_{n} \\
& \quad=\sum_{n=p+1}^{\infty}[(n-p)+\mu(n \beta-\alpha p)] \frac{n(k) \mu p(\beta-\alpha) \xi_{n}}{[(n-p)+\mu(n \beta-\alpha p)] n(k)} \\
& \quad \leq \mu p(\beta-\alpha) \sum_{n=p+1}^{\infty} \xi_{n} \leq \mu p(\beta-\alpha),
\end{aligned}
$$

since $0 \leq \sum_{n=p+1}^{\infty} \xi_{n} \leq 1$. Hence $f(z) \in S^{* *}(\alpha, \beta)$.

Conversly, suppose that $f(z)=z^{p}-\sum_{n=p+1}^{\infty} a_{n} z^{n} \in S^{* *}(\alpha, \beta)$. Therefore we have, for $n=2,3,4, \cdots$

$$
a_{n} \leq \frac{\mu p(\beta-\alpha)}{[(n-p)+\mu(n \beta-\alpha p)] n(k)}
$$

Setting $\xi_{n}=\frac{[(n-p)+\mu(n \beta-\alpha p)] n(k)}{\mu p(\beta-\alpha)} a_{n}$ for $n=2,3,4, \cdots$ and $\xi_{1}=1-$ $\sum_{n=p+1}^{\infty} \xi_{n}$, we find that $\xi_{n} \geq 0$ for $n=1,2,3, \cdots$

$$
\sum_{n=p+1}^{\infty} \xi_{n}=\sum_{n=p+1}^{\infty} \frac{[(n-p)+\mu(n \beta-\alpha p)] n(k)}{\mu p(\beta-\alpha)} a_{n} \leq 1
$$

since $f(z) \in S^{* *}(\alpha, \beta)$. And so $\xi_{1}=1-\sum_{n=1}^{\infty} \xi_{n} \geq 0$. Thus $\xi_{n} \geq 0$ for 


$$
\begin{aligned}
n=1,2,3, \cdots & \text { and } \sum_{n=1}^{\infty} \xi_{n}=1 \text {. Now } \\
f(z) & =z^{p}-\sum_{n=p+1}^{\infty} a_{n} z^{n} \\
& =z^{p}-\sum_{n=p+1}^{\infty} \frac{\mu p(\beta-\alpha)}{[(n-p)+\mu(n \beta-\alpha p)] n(k)} \xi_{n} z^{n} \\
& =\sum_{n=1}^{\infty} \xi_{n} f_{n}(z) .
\end{aligned}
$$

Hence we complete the proof of theorem. The proof of the Theorem 2.4 follows on lines similar to the proof of theorem on extreme points given in Silverman $([8])$.

Theorem 2.5(Closure Theorem). Let the function $f_{j}(z)$ defined by

$$
f_{j}(z)=z^{p}-\sum_{n=p+1}^{\infty} a_{n, j} z^{n}, \quad a_{n, j} \geq 0, \quad j=1,2,3 \cdots m
$$

be in the class $S^{* *}(\alpha, \beta)$. Then the function

$$
h(z)=z^{p}-\frac{1}{m} \sum_{n=p+1}^{\infty}\left(\sum_{j=1}^{m} a_{n, j}\right) z^{n}
$$

is in the class $S^{* *}(\alpha, \beta)$, where $\alpha=\min _{1 \leq j \leq m}\left\{\alpha_{j}\right\}, \quad-1 \leq \alpha_{j}<1$.

Proof. Since $f_{j}(z) \in S^{* *}(\alpha, \beta)$ for $j=1,2,3 \cdots m$, by Theorem 2.2 
we have

$$
\begin{aligned}
& \sum_{n=p+1}^{\infty}[(n-p)+\mu(n \beta-\alpha p)] n(k)\left(\frac{1}{m} \sum_{j=1}^{m} a_{n, j}\right) \\
& \quad=\frac{1}{m} \sum_{j=1}^{m}\left(\sum_{n=p+1}^{\infty}[(n-p)+\mu(n \beta-\alpha p)] n(k) a_{n, j}\right) \\
& \leq \frac{1}{m} \sum_{j=1}^{m} \mu p(\beta-\alpha j) \leq \mu p(\beta-\alpha) .
\end{aligned}
$$

Hence $h(z) \in S^{* *}(\alpha, \beta)$.

Next we prove the theorem for the radius of starlikeness and convexity.

THEOREM 2.6.

Let $f(z) \in S^{* *}(\alpha, \beta)$. Then

i) $f(z)$ is starlike of order $\delta(0 \leq \delta<1)$ in the disc

$$
|z| \leq r=\inf _{n \geq p+1}\left\{\frac{[(n-p)+\mu(n \beta-\alpha p)](2-p-\delta) n(k)}{\mu p(\beta-\alpha)(2-n-\delta)}\right\}^{\frac{1}{n-p}}
$$

ii) $f(z)$ is convex of order $\delta(0 \leq \delta<1)$ in the disc

$$
|z| \leq r=\inf _{n \geq p+1}\left\{\frac{p[(n-p)+\mu(n \beta-\alpha p)](2-p-\delta) n(k)}{n \mu p(\beta-\alpha)(2-n-\delta)}\right\}^{\frac{1}{n-p}}
$$

where $n=2,3, \cdots, p \in N$. These results are sharp for the function

$$
f(z)=z^{p}-\frac{\mu p(\beta-\alpha)}{[(n-p)+\mu(n \beta-\alpha p)] n(k)} z^{n} \quad(n=2,3, \cdots) .
$$


Proof. i) $f(z)$ is starlike of order $\delta(0 \leq \delta<1)$, we have $\operatorname{Re}\left\{\frac{z f^{\prime}(z)}{f(z)}\right\}>$ $\delta$. That is $\left|\frac{z f^{\prime}(z)}{f(z)}-1\right|<1-\delta$. Now

$$
\begin{aligned}
\left|\frac{z f^{\prime}(z)}{f(z)}-1\right| & \leq\left|\frac{(p-1) z^{p}-\sum_{n=p+1}^{\infty}(n-1) a_{n} z^{n}}{z^{p}-\sum_{n=p+1}^{\infty} a_{n} z^{n}}\right| \\
& <1-\delta \\
& \leq\left|(p-1) z^{p}-\sum_{n=p+1}^{\infty}(n-1) a_{n} z^{n}\right| \\
& <(1-\delta)\left|z^{p}-\sum_{n=p+1}^{\infty} a_{n} z^{n}\right| .
\end{aligned}
$$

Hence

$$
\sum_{n=p+1}^{\infty}\left(\frac{2-n-\delta}{2-p-\delta}\right) a_{n}|z|^{n-p}<1
$$

We note that $f(z) \in S^{* *}(\alpha, \beta)$ if and only if

$$
\sum_{n=p+1}^{\infty} \frac{[(n-p)+\mu(n \beta-\alpha p)] n(k) a_{n}}{\mu p(\beta-\alpha)}<1
$$

Using (2.7) and (2.8) we get

$$
\left(\frac{2-n-\delta}{2-p-\delta}\right)|z|^{n-p}<\frac{[(n-p)+\mu(n \beta-\alpha p)] n(k)}{\mu p(\beta-\alpha)} .
$$

Thus

$$
|z| \leq r=\inf _{n \geq p+1}\left\{\frac{[(n-p)+\mu(n \beta-\alpha p)](2-p-\delta) n(k)}{\mu p(\beta-\alpha)(2-n-\delta)}\right\}^{\frac{1}{n-p}}
$$

for $n=2,3,4, \cdots, p \in N$, which proves starlikeness of family. 
ii) $f(z)$ is convex of order $\delta(0 \leq \delta<1)$, we have $\operatorname{Re}\left\{1+\frac{z f^{\prime \prime}(z)}{f^{\prime}(z)}\right\}>$ $\delta$, that is

$$
\left|\frac{z f^{\prime \prime}(z)}{f^{\prime}(z)}\right|<1-\delta
$$

Thus

$$
\left|\frac{p(p-1) z^{p-1}-\sum_{n=p+1}^{\infty} a_{n} n(n-1) z^{n-1}}{p z^{p-1}-\sum_{n=p+1}^{\infty} a_{n} n z^{n-1}}\right|<1-\delta,
$$

that is

$$
\sum_{n=p+1}^{\infty} \frac{n(2-n-\delta)}{p(2-p-\delta)} a_{n}|z|^{n-p}<1
$$

We note that $f(z) \in S^{* *}(\alpha, \beta)$ if and only if

$$
\sum_{n=p+1}^{\infty} \frac{[(n-p)+\mu(n \beta-\alpha p)] n(k)}{\mu p(\beta-\alpha)} a_{n}<1 .
$$

Using (2.10) and (2.11), we get

$$
\frac{n}{p}\left(\frac{2-n-\delta}{2-p-\delta}\right)|z|^{n-p}<\frac{[(n-p)+\mu(n \beta-\alpha p)] n(k)}{\mu p(\beta, \alpha)} .
$$

Thus

$$
|z| \leq r=\inf _{n \geq p+1}\left\{\frac{p[(n-p)+\mu(n \beta-\alpha p)](2-p-\delta) n(k)}{n \mu p(\beta-\alpha)(2-n-\delta)}\right\}^{\frac{1}{n-p}},
$$

for $n=2,3,4, \cdots, p \in N$ which proves convex property of the family. $\square$

\section{Partial sums}

In this we consider partial sums of functions in the class $S^{* *}(\alpha, \beta)$ and obtain sharp lower bounds for the ratios of real part of $f(z), f_{k}(z)$ and $f^{\prime}(z)$ to $f_{k}^{\prime}(z)$. Silverman $([8])$ and Silvia([9]) have studied the partial sums of analytic functions. 
Theorem 3.1. Let $f(z) \in S^{* *}(\alpha, \beta)$ be given by (1.1) and define the partial sums of $f_{1}(z)$ to $f_{k}(z)$ by $f_{1}(z)=z^{p}$ and for $k=2,3, \cdots$

$$
f_{k}(z)=z^{p}+\sum_{n=p+1}^{k} a_{n} z^{n} \text {. }
$$

If $\sum_{n=p+1}^{\infty} t_{n}\left|a_{n}\right| \leq 1$ and

$$
t_{n}=\frac{[(n-p)+\mu(n \beta-\alpha p)]}{\mu p(\beta-\alpha)} n(k),
$$

then $f(z) \in S^{* *}(\alpha, \beta)$.

Furthermore,

$$
\operatorname{Re}\left\{\frac{f(z)}{f_{k}(z)}\right\}>1-\frac{1}{t_{k+1}}
$$

and

$$
\operatorname{Re}\left\{\frac{f_{k}(z)}{f(z)}\right\}>\frac{t_{k+1}}{1+t_{k+1}},
$$

where $z \in U, k \in N$.

Proof. For the coefficient $t_{n}$ given by (3.2), it is not difficult to verify that,

$$
t_{n+1}>t_{n}>1 \text {. }
$$

Therefore we have

$$
\sum_{n=p+1}^{\infty}\left|a_{n}\right|+t_{k+1} \sum_{n=k+1}^{\infty}\left|a_{n}\right| \leq \sum_{n=p+1}^{\infty} t_{n}\left|a_{n}\right| \leq 1 .
$$

By using (3.2) and by setting

$$
\begin{aligned}
g_{1}(z) & =t_{k+1}\left\{\frac{f(z)}{f_{k}(z)}-\left(1-\frac{1}{t_{k+1}}\right)\right\} \\
& =1+\frac{t_{k+1} \sum_{n=k+1}^{\infty} a_{n} z^{n}}{z^{p}+\sum_{n=p+1}^{k} a_{n} z^{n}}
\end{aligned}
$$


and using (3.6), we find that for $z \in U$,

$$
\begin{aligned}
\left|\frac{g_{1}(z)-1}{g_{1}(z)+1}\right| & \leq \frac{t_{k+1} \sum_{n=k+1}^{\infty} a_{n} z^{n}}{2 z^{p}+2 \sum_{n=p+1}^{k} a_{n} z^{n}+t_{k+1} \sum_{n=k+1}^{\infty} a_{n} z^{n}} \\
& \leq \frac{t_{k+1} \sum_{n=k+1}^{\infty}\left|a_{n}\right|}{2+2 \sum_{n=p+1}^{k}\left|a_{n}\right|+t_{k+1} \sum_{n=k+1}^{\infty}\left|a_{n}\right|} \\
& \leq 1
\end{aligned}
$$

which really gives the assertion (3.3) of Theorem 3.1.

In order to see that $f(z)=z^{p}+\frac{z^{k+1}}{t^{k+1}}$ gives sharp result, we observe that for $z=r e^{i \pi / k}, \frac{f(z)}{f_{k}(z)}=1+\frac{z^{k}}{t_{k+1}} \rightarrow 1-\frac{1}{t_{k+1}}$ as $z^{-}$. Similarly if we take

$$
\begin{aligned}
g_{2}(z) & =\left(1+t_{k+1}\right)\left\{\frac{f_{k}(z)}{f(z)}-\frac{t_{k+1}}{1+t_{k+1}}\right\} \\
& =1+\frac{\left(1+t_{k+1}\right) \sum_{n=p+1}^{k} a_{n} z^{n}}{z^{p}+\sum_{n=p+1}^{\infty} a_{n} z^{n}}
\end{aligned}
$$

and making use of (3.6), we can deduce that

$$
\begin{aligned}
\left|\frac{g_{2}(z)-1}{g_{2}(z)+1}\right| & \leq \frac{\left(1+t_{k+1)} \sum_{n=p+1}^{k} a_{n} z^{n}\right.}{2 z^{p}+2 \sum_{n=p+1}^{\infty} a_{n} z^{n}+\left(1+t_{k+1}\right) \sum_{n=p+1}^{k} a_{n} z^{n}} \\
& \leq \frac{\left(1+t_{k+1}\right) \sum_{n=p+1}^{k}\left|a_{n}\right|}{2+2 \sum_{n=p+1}^{\infty}\left|a_{n}\right|+\left(1+t_{k+1}\right) \sum_{n=p+1}^{k}\left|a_{n}\right|}
\end{aligned}
$$

which leads us to the assertion (3.4) of Theorem 3.1. The bound in (3.4) is sharp for each $k \in N$ with the extremal function $f(z)$ given.

THEOREM 3.2. If $f(z)$ of the form (1.1) satisfies the condition (2.1) then,

$$
\operatorname{Re}\left\{\frac{f^{\prime}(z)}{f_{k}^{\prime}(z)}\right\} \geq 1-\frac{k+1}{t_{k+1}}
$$


C. onsider

$$
\begin{aligned}
g(z) & =t_{k+1}\left\{\frac{f^{\prime}(z)}{f_{k}^{\prime}(z)}-\left(1-\frac{k+1}{t_{k+1}}\right)\right\} \\
& =1+\frac{\frac{t_{k+1}}{k+1} \sum_{n=k+1}^{\infty} a_{n} n z^{n-1}}{p z^{p-1}+\sum_{n=p+1}^{k} a_{n} n z^{n-1}} .
\end{aligned}
$$

Now

$$
\left|\frac{g(z)-1}{g(z)+1}\right| \leq \frac{\frac{t_{k+1}}{k+1} \sum_{n=k+1}^{\infty} n\left|a_{n}\right|}{-2 p-2 \sum_{n=p+1}^{k} n\left|a_{n}\right|-\frac{t_{k+1}}{k+1} \sum_{n=k+1}^{\infty}\left|a_{n}\right|} .
$$

If

$$
\frac{\frac{t_{k+1}}{k+1} \sum_{n=k+1}^{\infty} n\left|a_{n}\right|}{-2 p-2 \sum_{n=p+1}^{k} n\left|a_{n}\right|-\frac{t_{k+1}}{k+1} \sum_{n=k+1}^{\infty} n\left|a_{n}\right|} \leq 1,
$$

then

$$
\frac{t_{k+1}}{k+1} \sum_{n=k+1}^{\infty} n\left|a_{n}\right| \leq-2 p-2 \sum_{n=p+1}^{k} n\left|a_{n}\right|-\frac{t_{k+1}}{k+1} \sum_{n=p+1}^{\infty} n\left|a_{n}\right|,
$$

that is

$$
-\frac{1}{p} \frac{t_{k+1}}{k+1} \sum_{n=k+1}^{\infty} n\left|a_{n}\right|-\frac{1}{p} \sum_{n=p+1}^{k} n\left|a_{n}\right| \leq 1 \text {. }
$$

Since the left hand size of (3.14) is bounded above by $\sum_{n=p+1}^{k} t_{n}\left|a_{n}\right|$, if

$$
-\frac{1}{p} \frac{t_{k+1}}{k+1} \sum_{n=k+1}^{\infty} n\left|a_{n}\right|-\frac{1}{p} \sum_{n=p+1}^{k} n\left|a_{n}\right| \leq \sum_{n=p+1}^{k} t_{n}\left|a_{n}\right|
$$

then

$$
\sum_{n=p+1}^{k}\left(t_{n}+\frac{n}{p}\right)\left|a_{n}\right|+\sum_{n=k+1}^{\infty}\left[t_{n}+\frac{n t_{k+1}}{p(k+1)}\right]\left|a_{n}\right| \geq 0 .
$$

The result is sharp for the extremal function $f(z)$. 
THEOREm 3.3. If $f(z)$ of the form (1.1) satisfies the condition (2.1) then,

$$
\operatorname{Re}\left\{\frac{f_{k}^{\prime}(z)}{f^{\prime}(z)}\right\} \geq \frac{t_{k+1}}{k+1+t_{k+1}}
$$

Proof. By setting

$$
\begin{aligned}
g(z) & =\left(k+1+t_{k+1}\right)\left\{\frac{f_{k}^{\prime}(z)}{f^{\prime}(z)}-\frac{t_{k+1}}{k+1+t_{k+1}}\right\} \\
& =1-\frac{\frac{t_{k+1}}{k+1} \sum_{n=k+1}^{\infty} a_{n} n z^{n-1}}{p z^{p-1}+\sum_{n=p+1}^{\infty} a_{n} n z^{n-1}} .
\end{aligned}
$$

Making use of (3.13), we deduce that

$$
\begin{aligned}
\left|\frac{g(z)-1}{g(z)+1}\right| & \leq\left|\frac{\frac{t_{k+1}}{k+1} \sum_{n=k+1}^{\infty} a_{n} n z^{n-1}}{2 p z^{p-1}+2 \sum_{n=p+1}^{\infty} a_{n} n z^{n-1}-\frac{t_{k+1}}{k+1} \sum_{n=p+1}^{\infty} a_{n} n z^{n-1}}\right| \\
& \leq \frac{\frac{t_{k+1}}{k+1} \sum_{n=k+1}^{\infty} n\left|a_{n}\right|}{2 p+2 \sum_{n=p+1}^{\infty} n\left|a_{n}\right|-\frac{t_{k+1}}{k+1} \sum_{n=k+1}^{\infty} n\left|a_{n}\right|} \\
& \leq 1 .
\end{aligned}
$$

The result is sharp for the function $f(z)$.

\section{References}

[1] G. Murugusundaramoorthy, T. Rosy and K. Muthunagai,, Carlson-Shaffer Operator and their Applications to Certain Subclass of Uniformly Convex Functions, General Mathematics, Gen. Math. 15(04) (2007), 131-143.

[2] G. Ghanim and M. Darus, A New Subclass of Uniformly Starlike and Covex Functions with Negative Coefficients, Int. Math. Forum 4(23) (2009), 11051117.

[3] A. W. Goodman, On Uniformly Starlike Function, J. Math Anal. Appl. 155 (1991), 364-370.

[4] A. W. Goodman, On Uniformly Convex Function, Ann. Polon. Math. 56 (1991), 87-92.

[5] F. Ronning, Integral Representation for Bounded Starlike Functions, Ann. Polon. Math. 60 (1995), 289-297.

[6] F. Ronning, Unifromly Convex Functions and a corresponding Class of Starlike Functions, Proc. Amer. Math. Soc. 118 (1993), 189-196. 
[7] E. Aqlan, J. M. Jahangiri and S. R. Kulkarni, Classes of K-uniformly Convex and starlike Functions, Tamkang J. Math. 35(3) (2004), 261-266.

[8] H. Silverman, Partial-Sums of Starlike and Convex Functions, J. Math Anal. Appl. 209 (1997), 221-227.

[9] E. M. Silvia, Partial Sums of Convex Functions of order $\alpha$, Honston J. Math. Soc. 11(3) (1985), 397-404.

[10] K. G. Subramanian, G. Murugusundaramoorthy, P. Balasubrahmanyam and H. Silverman, Subclasses of Uniformly Convex and Uniformly starlike functions, Math. Japon. 42(3) (1995), 517-522.

[11] K. G. Subramanian, T. V. Sudharsan, P. Balasubrahmanyam and H. Silverman, Classes of Uniformly Starlike Functions, Publ. Math. Debrecen 53(34) (1998), 180-186.

[12] H. Silverman, Univalent Functions with negative coefficients, Proc. Amer. Math. Soc. 51 (1975), 109-116.

[13] B. C. Carlson and S. S. Shaffer, Starlike and Prestarlike Hypergeometric Functions, SIAM J. Math. Anal. 78 (1986), 737-745.

Department of Mathematics

and Education Research Institute

Gyeongsang National University

Jinju 660-701, Korea.

E-mail: sklee@gnu.ac.kr

Department of Mathematics

Maharashtra Academy of Engineering

Alandi, Pune - 412105

Maharashtra, India.

E-mail: smkhairnar2007@gmail.com

Department of Mathematics

G. H. Raisoni Institute of Engineering \& Technology

Pune - 412207, Maharashtra, India.

E-mail: sachin_rajas@rediffmail.com 